\title{
Screening for latent tuberculosis infection: performance of tuberculin skin test and interferon- $\gamma$ release assays under real-life conditions
}

\author{
S Kleinert, ${ }^{1}$ H-P Tony, ${ }^{1}$ K Krueger, ${ }^{2}$ J Detert, ${ }^{3}$ F Mielke, ${ }^{4}$ K Rockwitz, ${ }^{5}$ R Schwenke, ${ }^{6}$ \\ G R Burmester, ${ }^{3} \mathrm{R}$ Diel, ${ }^{7} \mathrm{M}$ Feuchtenberger, ${ }^{1} \mathrm{C}$ Kneitz ${ }^{8}$
}

- Additional tables are published online only. To view the files please visit the journal online (http://ard.bmj.com/ content/early/recent)

${ }^{1}$ Rheumatology/Clinical Immunology, University Hospital of Wuerzburg, Wuerzburg, Germany

2Praxiszentrum St Bonifatius München, München, Germany ${ }^{3}$ Department of Rheumatology and Clinical Immunology,

Charité - Universitätsmedizin

Berlin, a joint institution of the

Freie Universität Berlin and

the Humboldt - Universität zu, Berlin, Germany

${ }^{4}$ Gemeinschaftspraxis Innere Medizin, Dres Mielke, Berlin,

Germany

${ }^{5}$ Schwerpunktpraxis Innere

Medizin/Rheumatologie/

Osteologie, Goslar, Germany

${ }^{6}$ Schwerpunktpraxis Innere

Medizin/Rheumatologie/

Osteologie, Dresden, Germany

7Department of Pulmonary

Medicine, Hannover Medical

School, Hannover, Germany

${ }^{8}$ Klinikum Südstadt Rostock,

Klinik für Innere Medizin II,

Rostock, Germany

\section{Correspondence to}

Stefan Kleinert, Medizinische

Klinik und Poliklinik II,

Universitätsklinikum Würzburg,

Rheumatologie/Klinische Immunologie, Oberdürrbacherstr 6, 97080, Würzburg, Germany; Kleinert_S@klinik.uni-wuerzburg. de

Received 1 March 2012

Accepted 8 April 2012

Published Online First

14 May 2012

\begin{abstract}
Objectives To characterise optimal screening strategies for latent tuberculosis infection (LTBI) prior to the initiation of anti-tumour necrosis factor therapy.
\end{abstract}

Methods Patients in 62 German rheumatology centres were evaluated for LTBI. Each patient was screened with a tuberculin skin test (TST) and one form of an interferon- $\gamma$ release assay (IGRA), either TSPOT.TB (TSPOT) or Quantiferon TB Gold (QFT).

Results A total of 1529 patients with rheumatological disease were tested with a TST, 844 with TSPOT and 685 with OFT. TST was positive in $11.3 \%(n=173)$. The prevalence of $\mathrm{LTBI}$ was $8.0 \%$ when defined as a positive TST and no previous Bacille Calmette-Guérin (BCG) vaccination and $7.9 \%$ when based on a positive IGRA. Combining both estimates increased the prevalence of LTBI to $11.1 \%$. Clinical risk factors for LTBI were found in 122 patients (34 with a history of prior TB, 81 close contacts and 27 with suggestive chest $x$-ray lesions). A compound risk factor (CRF) was defined as the presence of at least one of these three risk factors. Statistical analyses were conducted to examine the association between CRF and LTBI test outcomes. In multivariate analysis, TST was influenced by CRF (OR 6.2; Cl 4.08 to $9.44, p<0.001$ ) and $B C G$ vaccination status (OR 2.9; $\mathrm{Cl} 2.00$ to 4.35, $\mathrm{p}<0.001)$. OFT and TSPOT were only influenced by CRF (QFT: OR 2.6; $\mathrm{Cl} 1.15$ to 5.98 , $p=0.021$; TSPOT: OR 8.7; Cl 4.83 to 15.82, $p<0.001$ ). ORs and the agreement of TST and IGRA test results varied by rheumatological disease.

Conclusion LTBI test results in an individual patient need to be considered in the context of prior BCG vaccination and clinical risk factors. In patient populations with low rates of TB incidence and BCG vaccination, the use of both TST and IGRA may maximise sensitivity in detecting LTBI but may also reduce specificity.

\section{INTRODUCTION}

The introduction of tumour necrosis factor (TNF) antagonists was a milestone towards better disease control in rheumatoid arthritis (RA). However, it was soon evident that TNF inhibitor therapy was associated with an increased risk of reactivation of latent tuberculosis infection (LTBI). ${ }^{1}$ National guidelines regarding screening for LTBI were established based on patients' history, clinical examination, chest $x$-ray and tuberculin skin test (TST) results. The effectiveness of recommendations based on
TST testing was proved by a Spanish cohort of patients treated with anti-TNF agents. ${ }^{2}$ Cases of TB reactivation that occurred in this cohort after establishment of recommendations were mainly due to non-adherence to recommendations. ${ }^{3}$

Interferon- $\gamma$ release assays (IGRAs) have shown superior results to TST in screening after TB exposure. ${ }^{4}$ Some national guidelines have recently favoured IGRA testing over TST for detection of LTBI. $^{5-7}$ The US Centers for Disease Control suggest using IGRA and TST if risk for progression to active tuberculosis is increased. ${ }^{8}$ Nevertheless, there are still open questions concerning the performance of different test systems in immunocompromised patients, such as patients with rheumatic diseases on immunosuppressive therapy, ${ }^{9}$ particularly with respect to the performance of IGRAs under real-life settings in daily practice. We therefore conducted a multicentre study to compare the utility of IGRA and TST in LTBI screening in a large cohort of patients with rheumatic diseases receiving immunosuppressive therapy.

\section{METHODS}

\section{Study design and patients}

This was a prospective study in which patients eligible for anti-TNF treatment were successively screened for LTBI at 62 participating centres located throughout Germany according to standard of care and national recommendations. Ethical approval for data management was obtained from the Ethics Committee of the Charité - Universitätsmedizin Berlin and approval for secure data management procedures was obtained from the data protection officer.

\section{LTBI screening}

Patients were screened for LTBI according to national guidelines. The patient's history of prior TB and close contact with TB patients was noted and Bacille Calmette-Guérin (BCG) vaccination status was documented. All patients underwent physical examination. Chest x-rays were obtained from 1409 patients. Interpretation regarding pulmonary lesions suggestive of latent or prior TB was based on the judgement of the local radiologist.

All patients received a TST and one type of IGRA, either TSPOT.TB (TSPOT) or Quantiferon TB Gold (QFT), depending on what was available in the corresponding laboratory. In accordance 
with contemporary guidelines for immunosuppressed patients, a TST with a diameter of $>5 \mathrm{~mm}$ skin induration was considered positive. The cut-off for TSPOT positivity was $\geq 6$ spots. At the time of testing, IGRAs were mainly based on the two peptide antigens ESAT-6 and CFP-10.

\section{Statistical analyses}

Clinical risk factors for LTBI were defined as a history of prior TB (prior TB), close contact to a patient with TB (contact) or a chest $\mathrm{x}$-ray suggestive of LTBI (CXR). A compound risk factor (CRF) was defined as the presence of at least one of these three risk factors.

Statistical analyses were performed using SPSS V.17.02 for Windows (SPSS, Chicago, Illinois, USA). Univariate regression analyses were used to calculate the influence of single risk factors; multivariate analyses used the variables CRF and BCG vaccination status to estimate the influence on test results. The results were reported as OR with CI. Cohen's $\kappa$ was calculated for test agreement (higher values indicate greater agreement).

Lymphocyte count and prednisone dose at the time of testing were documented in a subset of patients, and regression analyses were used to calculate the influence of these factors on $\mathrm{OR}$ for a negative or indeterminate test result.

\section{RESULTS}

\section{Patient characteristics}

A total of 1609 patients eligible for anti-TNF treatment were successively screened for LTBI. Eighty patients were excluded from the main analysis due to indeterminate IGRA results, leaving a total of 1529 patients. All of the patients had rheumatic diseases, including rheumatoid arthritis (RA; $n=852$, mean DAS28 4.35 \pm 1.88 , median disease duration 7.4 years), ankylosing spondylitis (AS; $\mathrm{n}=294$, median disease duration 7.5 years), psoriatic arthritis (PsA; $n=215$, median disease duration 6.9 years), undifferentiated spondyloarthropathy (SpA; $n=92$, median disease duration 3.6 years) and various other rheumatologic disorders ( $n=76$, median disease duration 1.3 years). More than half of the patients $(61.3 \%)$ were women. Of the 1529 patients, 204 $(13.3 \%)$ had been vaccinated with BCG. Patient characteristics and risk factors by TST and IGRA status are shown in figure 1.

\section{Estimation of LTBI prevalence}

Positive TSTs were recorded in $11.3 \%(n=173)$ of patients (figure 1). The prevalence of LTBI was $8.0 \%(n=123)$ as estimated by a positive TST in patients without previous BCG vaccination or $7.9 \%(n=120)$ based solely on a positive IGRA $(8.3 \%$ in the TSPOT group and $7.3 \%$ in the OFT group). The estimated prevalence of LTBI increased to $11.1 \%$ if LTBI was defined as meeting either of these criteria. Only $4.3 \%$ of patients $(n=66)$ were positive for both TST and IGRA.

\section{Influence of risk factors on test results}

Clinical risk factors for LTBI were found in 122 patients (34 with a history of prior TB, 81 close contacts and 27 with a chest x-ray suggestive of LTBI). A CRF was defined as the presence of at least one of these risk factors. Risk factors were more common in patients with positive LTBI screening results (table 1).

Statistical analyses were used to examine the impact of risk factors on screening results. For TST and TSPOT, all single risk factors influenced test results while, for OFT, only the risk factor prior TB had an influence on test results (see table S1 in online supplement).

We further analysed the influence of CRF and BCG vaccination status on the LTBI screening results. In multivariate analyses, patients with CRF had a 6.2-fold increased risk for positive TST in the total population (table 2). CRF was associated with a 2.6fold increased risk for a positive OFT and an 8.7-fold increased

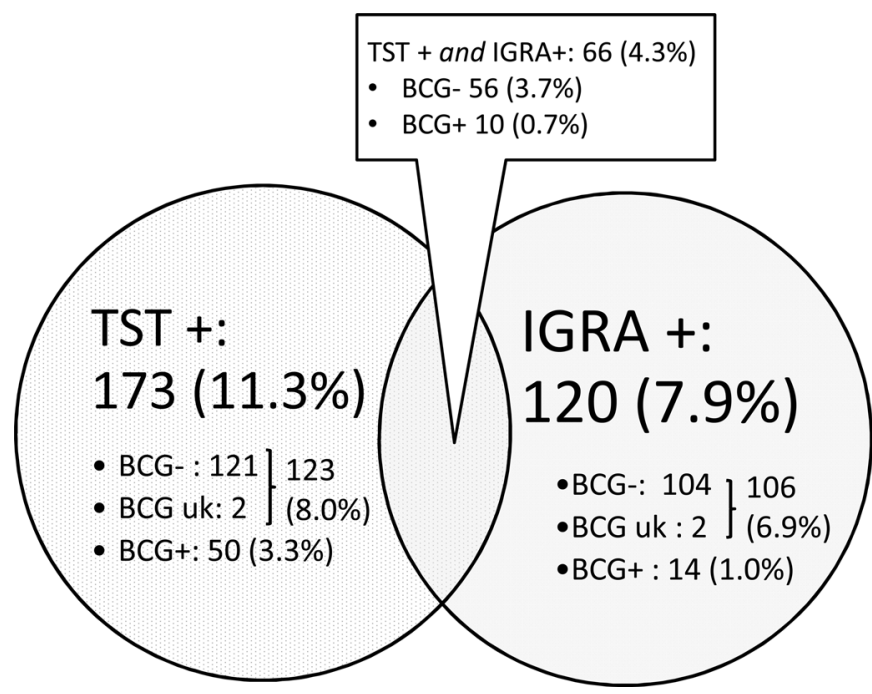

Figure 1 LTBI screening results in 1529 German rheumatology patients preparing to start treatment with tumour necrosis factor (TNF) inhibitors. TST+, tuberculin skin test positive; IGRA + , interferon- $\gamma$ release assay positive. BCG +/-/uk: BCG vaccinated/not vaccinated/ vaccination status unknown. Data represent patient numbers (\%).

Table 1 Age, BCG vaccination status and clinical risk factors by LTBI screening results

\begin{tabular}{|c|c|c|c|c|c|c|}
\hline \multirow[b]{2}{*}{ Parameter } & \multicolumn{2}{|c|}{ TST (n=1529) } & \multicolumn{2}{|c|}{ OFT $(n=685)$} & \multicolumn{2}{|c|}{ TSPOT $(n=844)$} \\
\hline & Positive (n=173) & Negative $(n=1356)$ & Positive $(n=50)$ & Negative $(n=635)$ & Positive $(n=70)$ & Negative $(n=774)$ \\
\hline Age, mean years $( \pm S D)$ & $52.0 \pm 12.1$ & $51.6 \pm 14.1$ & $58.4 \pm 12.7$ & $51.1 \pm 13.9$ & $59.5 \pm 12.9$ & $50.8 \pm 13.8$ \\
\hline $\mathrm{BCG}^{*}{ }^{*} \mathrm{n}(\%)$ & $50(28.9)$ & $154(11.4)$ & $5(10.0)$ & $127(20.0)$ & $9(12.9)$ & $63(8.1)$ \\
\hline Contact, $†$ n (\%) & $29(16.8)$ & $52(3.8)$ & $3(6.0)$ & $30(4.7)$ & $11(15.7)$ & $37(4.8)$ \\
\hline $\mathrm{CXR}, \mp \mathrm{n}(\%)$ & $15(8.7)$ & $12(0.9)$ & $3(6.0)$ & $7(1.1)$ & 15 (21.4) & $2(0.3)$ \\
\hline CRF, $†$ n (\%) & $48(27.7)$ & $74(5.5)$ & $9(18.0)$ & $45(7.1)$ & $24(34.3)$ & $44(5.7)$ \\
\hline
\end{tabular}

\footnotetext{
Data represent patient numbers (\%) positive for vaccination or stated risk factor unless otherwise indicated.

CRF was defined as the presence of at least one of the following three clinical risk factors: history of prior TB, close contact and chest X-ray suggestive of LTBI (CXR).

*Twenty-one patients had missing data on BCG vaccination ( 2 in the TST-positive group and 19 in the TST-negative group). Eighteen of the 21 patients were tested with QFT (1 positive, 17 negative) and the remaining 3 were tested with TSPOT (1 positive, 2 negative).

tFive patients had missing results on prior TB/contact and therefore also on CRF. All 5 were in the TST-negative group (2 in the QFT-negative group, 2 in the TSPOT-positive group and 1 in the TSPOT-negative group).

¥Chest $x$-ray was performed in $88.2-97.1 \%$ of patients in each group.

BCG, Bacille Calmette-Guérin; CRF, compound risk factor; LTBI, latent tuberculosis infection; QFT, Quantiferon TB Gold; TSPOT, TSPOT.TB; TST, tuberculin skin test.
} 
Table 2 Multivariate regression analyses of the effect of the CRF and BCG vaccination on $L T B I$ screening results

\begin{tabular}{|c|c|c|c|c|}
\hline Test & Risk factor & OR & $95 \% \mathrm{Cl}$ & p Value \\
\hline \multicolumn{5}{|c|}{ Patients tested with TST $(n=1503)^{*}$} \\
\hline \multirow[t]{2}{*}{ TST } & CRF & 6.20 & 4.08 to 9.44 & $<0.001$ \\
\hline & BCG & 2.95 & 2.00 to 4.35 & $<0.001$ \\
\hline \multicolumn{5}{|c|}{ Patients tested with QFT and TST $(n=667) \dagger$} \\
\hline \multirow[t]{2}{*}{ QFT } & CRF & 2.63 & 1.15 to 5.98 & 0.021 \\
\hline & BCG & 0.43 & 0.17 to 1.10 & 0.079 \\
\hline \multirow[t]{2}{*}{ TST } & CRF & 4.85 & 2.58 to 9.09 & $<0.001$ \\
\hline & BCG & 3.09 & 1.90 to 5.03 & $<0.001$ \\
\hline \multicolumn{5}{|c|}{ Patients tested with TSPOT and TST $(n=836) \ddagger$} \\
\hline \multirow[t]{2}{*}{ TSPOT } & CRF & 8.74 & 4.83 to 15.82 & $<0.001$ \\
\hline & BCG & 1.07 & 0.47 to 2.43 & 0.869 \\
\hline \multirow[t]{2}{*}{ TST } & CRF & 8.30 & 4.69 to 14.70 & $<0.001$ \\
\hline & BCG & 2.07 & 1.05 to 4.11 & 0.037 \\
\hline
\end{tabular}

*Excluding 5 patients with missing CRF results and 21 patients with missing BCG status.

†Excluding 2 patients with missing CRF results and 16 patients with missing BCG status.

łExcluding 3 patients with missing CRF results and 5 patients with missing BCG status BCG, Bacille Calmette-Guérin; CRF, compound risk factor; LTBI, latent tuberculosis infection; QFT, Quantiferon TB Gold; TSPOT, TSPOT.TB; TST, tuberculin skin test.

risk for a positive TSPOT. Univariate analyses revealed very similar results (data not shown). Test results were independent of the time interval between TST and IGRA ( $p=0.21$; data not shown).

\section{Influence of risk factors in disease subgroups}

Multivariate regression analyses of patients with RA $(n=852)$ showed that CRF influenced TST (OR 8.44, CI 4.71 to 15.14, $\mathrm{p}<0.001$ ) and TSPOT (OR 10.69, CI 5.01 to 22.81, $\mathrm{p}<0.001$ ) but not OFT (OR 0.69, CI 0.08 to 4.66, p=0.625). In patients with PsA ( $\mathrm{n}=215)$, CRF had no significant influence on TSPOT (OR 3.54, CI 0.56 to $22.33, p=0.179$ ) or TST (OR 1.98, CI 0.61 to 6.38, $p=0.253$ ) and could not be calculated for OFT due to the absence of patients with CRF and positive OFT. In patients with AS or SpA ( $n=385)$, CRF influenced TST (OR 7.88, CI 3.44 to 18.05, p<0.001), TSPOT (OR 7.03, CI 2.76 to 17.89, $\mathrm{p}<0.001$ ) and OFT (OR 7.29, CI 2.87 to 18.52, p<0.001). Complete disease subgroup data are shown in table S2 in the online supplement. Univariate results were very similar.

\section{Agreement between TST and IGRA}

The patients' characteristics in the concordant and discordant subgroups are shown in table 3. Concordance of TST and IGRA was $89.5 \%$ but Cohen's $\kappa$ was 0.40 ( $p<0.001)$, indicating that the agreement was only fair to moderate, although significant. Concordance was $87.6 \%(\kappa=0.34, \mathrm{p}<0.001)$ between TST and OFT and $91.1 \%(\kappa=0.44, p<0.001)$ between TST and TSPOT. Agreement was significant in the total population and all disease subgroups except those with PsA (see table S3 in online supplement). The subgroup of TST-positive patients with a skin induration $\geq 15 \mathrm{~mm}(\mathrm{n}=71)$ included 27 that were not BCG vaccinated and were IGRA-negative.

\section{Influence of BCG vaccination}

Of the 204 patients who were BCG vaccinated, 50 were TSTpositive and 14 were IGRA-positive (table 1). BCG status influenced TST but not OFT and TSPOT (table 2). Concordance was lower in the BCG vaccinated group $(79.9 \%,=0.27, \mathrm{p}<0.001)$ than in the non-vaccinated group $(\mathrm{n}=1303 ; 91.0 \%,=0.43, \mathrm{p}<0.001)$.
Table 3 Patient characteristics, BCG vaccination status and clinical risk factors by concordance or discordance group

\begin{tabular}{|c|c|c|c|c|}
\hline \multirow[b]{2}{*}{ Parameter } & \multicolumn{2}{|c|}{ Concordance } & \multicolumn{2}{|c|}{ Discordance } \\
\hline & $\begin{array}{l}\text { TST+/IGRA } \\
+(n=66)\end{array}$ & $\begin{array}{l}\text { TST-/ } \\
\text { IGRA-(n=1302) }\end{array}$ & $\begin{array}{l}\text { TST+/ } \\
\text { IGRA-(n=107) }\end{array}$ & $\begin{array}{l}\text { TST-/IGRA } \\
+(n=54)\end{array}$ \\
\hline $\begin{array}{l}\text { Age, mean } \\
\text { years } \\
( \pm S D)\end{array}$ & $57.9 \pm 11.0$ & $51.2 \pm 14.0$ & $48.4 \pm 11.4$ & $60.0 \pm 14.7$ \\
\hline $\begin{array}{l}\text { Female sex, } \\
\mathrm{n}(\%)\end{array}$ & $40(60.6)$ & $809(62.1)$ & $63(58.9)$ & $26(48.1)$ \\
\hline $\mathrm{BCG}^{*}{ }^{*} \mathrm{n}(\%)$ & $11(16.7)$ & $152(11.7)$ & $39(36.4)$ & $3(5.6)$ \\
\hline Prior $T B, \dagger \mathrm{n}$ & 12 (18.2) & $15(1.2)$ & $4(3.8)$ & $3(5.6)$ \\
\hline Contact, $\uparrow \mathrm{n}$ & 13 (19.7) & $52(4.0)$ & $16(15.0)$ & $1(1.9)$ \\
\hline $\mathrm{CXR}, \ddagger \mathrm{n}(\%)$ & 13 (19.7) & $7(0.5)$ & $2(1.9)$ & $5(9.3)$ \\
\hline CRF,† n (\%) & 27 (40.9) & $69(5.3)$ & 21 (19.6) & $6(11.1)$ \\
\hline
\end{tabular}

Data represent patient numbers (\%) positive for vaccination or stated risk factor unless otherwise indicated.

CRF was defined as the presence of at least one of the following three clinical risk factors: history of prior TB, close contact and chest x-ray suggestive of LTBI (CXR). *Twenty-one patients with missing BCG data were excluded from BCG analyses (18 in TST-/IGRA - and 1 each in the other 3 groups).

tFive patients with missing results for prior TB and close contact (1 in TST-/IGRA+, 4 in TST-/IGRA - ) were excluded from these analyses and from the CRF analysis. ҒCXR was performed in $86.0-96.3 \%$ of patients in each group.

BCG, Bacille Calmette-Guérin; CRF, compound risk factor; IGRA, interferon- $\gamma$ release assay; LTBI, latent tuberculosis infection; TST, tuberculin skin test.

\section{Influence of lymphocyte count and prednisone dose on test results}

In a subgroup analysis of 507 patients with data on prednisone dose and lymphocyte counts, ORs for negative or indeterminate test results were not affected by low lymphocyte count (compared with a normal lymphocyte count) or the use of prednisone (compared with non-use) (see table S4 in online supplement).

\section{DISCUSSION}

Testing for LTBI using the classical risk factors and TST has been proved to reduce the incidence of $\mathrm{TB}$ reactivation in patients treated with TNF inhibitors. ${ }^{2}$ IGRAs have shown superior results for screening after TB exposure ${ }^{4}$ but their utility in LTBI screening in everyday practice has not been well studied, particularly with respect to detecting LTBI and preventing TB reactivation in patients preparing to start anti-TNF treatment. Because there is no 'gold standard' for diagnosing LTBI, it is not possible to determine whether discrepancies among test systems reflect false positives or false negatives or to calculate the sensitivity and specificity of TB test systems. To gain a better understanding of optimal LTBI screening methods, we therefore compared the performance of TST and IGRA (either TSPOT or OFT) in a multicentre study of 1529 consecutive German patients with rheumatological disease tested for LTBI prior to anti-TNF treatment and analysed the impact of BCG vaccination and LTBI risk factors on screening results.

For our analysis of different LTBI screening systems in a country with a low incidence of TB, we used a positive TST without previous BCG vaccination or a positive IGRA as surrogate definitions for LTBI, although this is not recommended in clinical practice. In particular, in populations with high incidences of $\mathrm{TB}$, it is recommended that a positive TST in the absence of active TB should be classified as LTBI regardless of prior BCG vaccination. ${ }^{6}$

Our study revealed an LTBI prevalence of $8.0 \%$ based on TST/BCG vaccination status and $7.9 \%$ based on IGRA testing. When LTBI was defined as a positive response in either test system, the prevalence rate increased to $11.1 \%$. These prevalence 
rates are comparable to those from a German study in which an LTBI prevalence of $7.2 \%$ (defined by OFT testing) was reported among healthcare workers. ${ }^{10}$

Owing to the lack of a gold standard for LTBI testing, we calculated the association between positive tests results and known LTBI clinical risk factors (prior TB disease, contact history and chest $\mathrm{x}$-ray lesions suggestive of latent or prior $\mathrm{TB}$ ) to help assess the performance of these testing systems. All the risk factors influenced the test results for TST and TSPOT whereas, for QFT, only prior TB exerted an influence. CRF influenced the results for all three of the tests but had less influence on QFT than on the other test systems. It should be noted that our study was not designed to compare the two IGRAs (OFT and TSPOT) directly, so the correlation of clinical risk factors with test results provided the only means of estimating their relative utility. By this standard, TSPOT appears to perform better than QFT due to its greater correlation with known LTBI risk factors. Nevertheless, we cannot exclude the possibility that a poorer correlation with clinical risk factors is due to a higher specificity rather than a lower sensitivity. A better understanding of the relative merit of OFT versus TSPOT will require head-to-head tests under real-world conditions.

Important differences in the influence of risk factors on screening results and concordance of TST and IGRA tests were observed among disease subgroups. In the RA subgroup, OFT was not influenced by CRF. In the PsA subgroup, CRF did not influence any test and concordance of TST and IGRA was not significant whereas, in the SpA group, the influence of CRF on all tests was high. These discrepancies could indicate unknown confounders due to immunological disease characteristics or to the intensity and duration of previous immunosuppressive therapies. Our findings suggest that optimal LTBI screening strategies for patients with PsA may be particularly elusive.

A subgroup analysis of 507 patients with appropriate data did not reveal a statistically significant influence of prednisone use or lymphopenia on test results. Data on previous therapies and concomitant disease-modifying antirheumatic drugs were not recorded completely and therefore were not analysed.

Our study has some limitations that should be considered when evaluating the findings. The lack of a gold standard for LTBI diagnosis means that we cannot directly determine the sensitivity or specificity of the LTBI tests, nor were we able to obtain subsequent clinical information on the development of $\mathrm{TB}$ in these patients. In addition, the multicentre nature of our study, which involved the use of many different clinicians administering tests and laboratories assessing results, may add some unknown confounders but definitely resembles real-life conditions. Finally, these results were obtained in a country with a low incidence of TB (approximately four active TB cases per 100 000 according to 2010 World Health Organization figures) and low BCG vaccination rate (13.3\% in our study). Accordingly, the conclusions may not necessarily apply to countries with higher rates of either $\mathrm{TB}$ or vaccination.

The results from our study and others lend some support to the suggestion that IGRA may be preferable to TST when diagnosing LTBI in populations with a low incidence of TB and a high vaccination rate. ${ }^{6}$ A single-centre study of 142 Swiss patients with autoimmune diseases, $83 \%$ of whom had been vaccinated with BCG, reported a $12 \%$ positive rate for OFT and $32 \%$ for TST; the tests showed low concordance (64\%) with poor agreement $(\kappa=0.17)$, and risk factors exerted a much stronger influence on QFT than on TST. ${ }^{11}$ It is likely that the high vaccination rate reduced the specificity of TST in this population. Our study and others, including a pooled analysis of data from multicentre phase III studies of rheumatology patients $(\mathrm{n}=2282),{ }^{12}$ have shown higher concordances between TST and IGRA (87-89.5\%), but $\kappa$ values indicate only fair to moderate agreement (ranging from 0.22 in the pooled phase III studies with $38.5 \%$ BCG vaccinated to 0.40 in our study with $13.3 \%$ BCG vaccinated). Furthermore, our data indicate that the concordance of TST and IGRA is lower in the BCG vaccinated group. As expected, IGRA test results were not influenced by prior BCG vaccination, suggesting a higher specificity for diagnosing LTBI in these patients. The higher specificity in BCG vaccinated patients and the ease in doing a blood test without requiring the patient to return to the clinician's office, as for skin tests, has contributed to the success of IGRA.

Although IGRA may have some advantages over TST in certain settings (eg, populations with high vaccination rates), our study also raises important concerns about this testing method for patients with rheumatic diseases evaluated prior to biological therapy. Although LTBI testing based on TST has been proved to be effective in patients with rheumatological diseases prior to anti-TNF treatment, ${ }^{2}$ in our study there was only a partial overlap in the populations judged to have LTBI based on TST and IGRA. Special concern needs to be raised regarding the subgroup of 71 TST-positive patients with a skin induration $\geq 15 \mathrm{~mm}$, usually considered a sign of true infection. Of this group, 27 (38\%) were IGRA-negative and had not been vaccinated with BCG. This finding suggests that the real-world effectiveness of IGRA in diagnosing LTBI and therefore preventing TB reactivation in patients prior to biological therapy requires further study. TST may have the additional benefit of detecting latent infection with atypical mycobacteria whereas IGRA does not. However, the impact of anti-TNF treatment on these infections needs further elucidation.

We conclude that, in patient populations with low rates of TB incidence and BCG vaccination, employing both TST and IGRA may maximise sensitivity in detecting LTBI but may also reduce specificity. From the clinician's perspective, it is important to bear in mind that false positive or false negative tests can occur with either IGRA or TST. Accordingly, test results in each individual patient should be considered in the context of BCG vaccination status and probable LTBI risk as defined by clinical risk factors.

Acknowledgments The authors would like to thank all participating centres for their contribution to the study, Dr Imma Fischer, Tuebingen, for statistical analysis and Sharon L Cross for reviewing the final manuscript.

Competing interests SK and CK received a research grant from Abbott. SK has received speakers' fees from Abbott, Chugai, Roche and Essex. HPT has received speakers' or consulting fees from Abbott, Roche and Wyeth and a research grant from Chugai. GRB has received research grants, consulting and speakers' fees from Abbott. RD has received travel reimbursement and/or fees for speaking at symposia sponsored by Cellestis Ltd., Oxford Immunotec Ltd. and Pharmore Ltd. (exclusive supplier of Tuberculin RT23 for Germany). CK has received speakers' or consulting fees from Abbott, Chugai, MSD, Oxford Immunotec, Roche and Pfizer. JD has received research grants from Abbott, Pfizer.

Funding The work was funded by an unrestricted grant from Abbott $\mathrm{GmbH}$ used to support the conduct of the study, data analysis and editing and review of the manuscript.

Provenance and peer review Not commissioned; externally peer reviewed.

Correction notice This article has been corrected since it was published Online First.

\section{REFERENCES}

1. Keane J, Gershon S, Wise RP, et al. Tuberculosis associated with infliximab, a tumor necrosis factor alpha-neutralizing agent. N Engl J Med 2001;345:1098-104.

2. Carmona L, Gómez-Reino JJ, Rodríguez-Valverde V, et al. Effectiveness of recommendations to prevent reactivation of latent tuberculosis infection in patients treated with tumor necrosis factor antagonists. Arthritis Rheum 2005;52:1766-72. 
3. Gómez-Reino JJ, Carmona L, Angel Descalzo M. Risk of tuberculosis in patients treated with tumor necrosis factor antagonists due to incomplete prevention of reactivation of latent infection. Arthritis Rheum 2007:57:756-61.

4. Diel R, Loddenkemper R, Meywald-Walter K, et al. Comparative performance of tuberculin skin test, QuantiFERON-TB-Gold In Tube assay, and T-Spot.TB test in contact investigations for tuberculosis. Chest 2009;135:1010-8.

5. Diel R, Hauer B, Loddenkemper R, et al. [Recommendations for tuberculosis screening before initiation of TNF-alpha-inhibitor treatment in rheumatic diseases]. Z Rheumatol 2009;68:411-6.

6. Solovic I, Sester M, Gomez-Reino JJ, et al. The risk of tuberculosis related to tumour necrosis factor antagonist therapies: a TBNET consensus statement. Eur Respir J 2010;36:1185-206.

7. Smith R, Cattamanchi A, Steingart KR, et al. Interferon-gamma release assays for diagnosis of latent tuberculosis infection: evidence in immune-mediated inflammatory disorders. Curr Opin Rheumatol 2011;23:377-84.

8. Mazurek GH, Jereb J, Vernon A, et al. Updated guidelines for using Interferon Gamma Release Assays to detect Mycobacterium tuberculosis infection - United States, 2010. MMWR Recomm Rep 2010;59:1-25.
9. Kleinert S, Kurzai 0, Elias J, et al. Comparison of two interferon-gamma release assays and tuberculin skin test for detecting latent tuberculosis in patients with immune-mediated inflammatory diseases. Ann Rheum Dis 2010;69:782-4.

10. Schablon A, Beckmann G, Harling M, et al. Prevalence of latent tuberculosis infection among health care workers in a hospital for pulmonary diseases. J Occup Med Toxicol 2009; 4:1.

11. Matulis G, Jüni P, Villiger PM, et al. Detection of latent tuberculosis in immunosuppressed patients with autoimmune diseases: performance of a Mycobacterium tuberculosis antigen-specific interferon gamma assay. Ann Rheum Dis 2008;67:84-90.

12. Hsia EC, Schluger N, Cush J, et al. Interferon-gamma release assay versus tuberculin skin test across RA, PsA, and AS patients prior to treatment with golimumab, a human anti-TNF antibody. Arthritis Rheum 20122012 Jan 11. doi: 10.1002/art.34382. [Epub ahead of print] 